\title{
Effects of silver sulphadiazine on the production of exoproteins by Staphylococcus aureus
}

\author{
VALERIE EDWARDS-JONES and H. A. FOSTER* \\ Department of Biological Sciences, Manchester Metropolitan University, Chester Street, Manchester M1 5 GD \\ and *Division of Biological Sciences, School of Environment and Life Sciences, University of Salford, The \\ Crescent, Salford, Lancs M5 4WT
}

\begin{abstract}
The effects of subinhibitory concentrations of silver sulphadiazine (AgSD) on exoprotein production in Staphylococcus aureus strains T1, T4, RN4282 and RN 4282agr were studied. AgSD markedly increased levels of toxic shock syndrome toxin (TSST)-1 in strains T4 and RN4282. This effect was independent of agr and AgSD restored TSST-1 production to the wild-type level in RN 4282agr. AgSD had no effect on enterotoxin A or coagulase activity in strains $T 1$ or $T 4$. Strain $T 4$ produced enterotoxin $C$ at high levels and no effect was observed with AgSD. AgSD repressed metalloprotease production in strain $\mathrm{T} 4$ but the overall protease activity remained the same. No change in proteolytic activities was seen in strain T1 with AgSD. Molecular mechanisms for these observations are discussed.
\end{abstract}

\section{Introduction}

Staphylococcus aureus is the most common cause of nocosomial infection and is of increasing concern because of the spread of methicillin-resistant strains and the occurrence of strains additionally showing resistance to vancomycin [1]. It is a pathogen that is capable of causing various diseases from mild skin infections through to life-threatening diseases such as toxic shock syndrome (TSS) [2]. The pathogenicity has been attributed to the ability of the organism to elaborate a large number of surface bound proteins, e.g., protein A, adhesins and secreted toxins, e.g., enterotoxins, exfoliative toxins, toxic shock syndrome toxin (TSST)-1. Other exoproteins are involved in the infection process, e.g., proteolytic enzymes, haemolysins, coagulase, lipase and deoxyribonuclease [3, 4]. The production of these virulence determinants is tightly controlled and is dependent on the phase of growth, cell density, metal ions and physiological conditions [5-7]. They are regulated by a number of global regulons including agr and sar [8,9]. These contribute to the upregulation of exoproteins and the down-regulation or modification of surface-bound proteins in response to cell density and environmental stress, e.g., microaerobic conditions $[6,9]$.

Received 14 April 2000; revised version received 10 July 2001; accepted 14 July 2001.

Corresponding author: Dr H. A. Foster (e-mail: h.a.foster@ salford.ac.uk).
Antimicrobial agents can also affect the production of toxins such as TSST-1 at concentrations that do not affect growth. Clindamycin, gentamicin and macrolides such as erythromycin inhibit toxin synthesis [10], whereas $\beta$-lactams stimulate synthesis [11]. Glycerol monolaurate also inhibits TSST-1 synthesis $[12,13]$.

A previous report showed that the production of TSST1 was affected by subinhibitory concentrations of topical antimicrobial preparations. TSST-1 production was increased by $4-16$-fold in $45 \%$ of strains by exposure to Flamazine ${ }^{\mathrm{TM}}$ [14] which contains silver sulphadiazine (AgSD) as the active ingredient. Silver sulphanilamides were first synthesised in 1942 [15] but it was not until 1968 that $\mathrm{AgSD}$ was introduced for prevention and treatment of infection of burn wounds [16]. It has since been widely accepted as being very effective $[17,18]$. AgSD is relatively insoluble and it dissociates slowly to give a sustained release of silver ions into the wound environment $[18,19]$. The sulphonamide moiety is at a concentration below that required for antimicrobial action [20]. There is no cross-reaction between sulphonamide resistance and sensitivity to AgSD but sulphonamide-resistant $S$. aureus are killed more slowly than sulphonamidesensitive strains [21,22]. Use of AgSD can also increase the incidence of sulphonamide resistance on a burns unit [22], which suggests that the sulphadiazine is having some effect. Sulphadiazine potentiates the action of AgSD and it has been suggested that this may be due to a reduced degree of ionisation of the $\mathrm{AgSD}$, 
thus slowing the release of Ag into the medium [18]. Although AgSD interacts irreversibly with DNA to form a complex in vitro [23], this does not appear to be its mode of action in vivo and $\mathrm{Ag}^{2+}$ ion is the active agent [24]. Silver ions react with DNA in vitro, firstly in a weak interaction then strongly with the silver ions becoming incorporated by bonding between the base pairs [25]. Although it has been suggested that binding to DNA is the mode of action in vivo [18], other workers suggest that $\mathrm{Ag}^{2+}$ binds preferentially to external cell structures and that only small amounts are bound to DNA [24]. Cells of $S$. aureus treated with AgSD were enlarged, some retraction of the cytoplasmic membrane from the cell wall was seen and division planes between bacteria became indistinct, supporting the view that the effect is on external cell structures [26]. Studies have shown that subinhibitory levels of sulphadiazine affect membrane permeability [27]. This may enhance the entry of silver ions into cells. This study determined which component of Flamazine ${ }^{\mathrm{TM}}$ preparations was responsible for the effects on TSST-1 production and the effects of $\mathrm{AgSD}$ on other virulence factors were investigated.

\section{Materials and methods}

\section{Bacterial strains and culture conditions}

S. aureus strains T1 and T4 were as described previously [14]. Strain T1 (FRI1189S) was isolated from a case of menstrual TSS (MTSS) and originally supplied by M. S. Bergdoll. Strain T4 was originally isolated from a burned patient with confirmed TSS (Bury and Rochdale Healthcare Trust). Strain T1 showed no response to Flamazine ${ }^{\mathrm{TM}}$ whereas strain $\mathrm{T} 4$ produced at least eight-fold more TSST-1 in subinhibitory concentrations. Strains RN4282 and RN4282agr were supplied by T. J. Foster, University of Dublin, and were originally from R. P. Novick. Cultures were grown in $50 \mathrm{ml}$ of Brain-Heart Infusion Broth (BHI, Oxoid) in $100-\mathrm{ml}$ conical flasks in an orbital incubator at $37^{\circ} \mathrm{C}$ and $200 \mathrm{rpm}$ for $24 \mathrm{~h}$. Culture supernates were obtained by centrifugation at $1500 \mathrm{~g}$ for $20 \mathrm{~min}$ and the supernatant fluids were removed and stored at $-70^{\circ} \mathrm{C}$.

\section{Viable counts}

Samples were serially diluted in saline and samples were inoculated on to Nutrient Agar plates (Oxoid) by the method of Miles and Misra [28].

\section{Determination of TSST-1 and enterotoxins}

TSST-1 and enterotoxins A and C (SEA and SEC) in culture supernatant fluids were determined with reverse passive latex agglutination kits supplied by Unipath (Oxoid TD 40 and TD900) used according to the manufacturer's instructions. Briefly, the supernates were double diluted in PBS supplied with the kit. This was mixed with an equal volume $(25 \mu \mathrm{l})$ of sensitised latex particles and allowed to react at room temperature. The titre was determined as the last dilution showing agglutination. Hence the results were semi-quantitative and assumed that this titre represented a concentration of $2 \mathrm{ng} / \mathrm{ml}$. Kits from the same batch were used within each experiment to ensure comparability. TSST-1 was also expressed as $\mathrm{ng} / \mathrm{ml} / 10^{9}$ cells (specific toxin production).

\section{Determination of the active components of Flamazine $^{T M}$}

Subinhibitory concentrations were chosen that allowed comparison of growth and toxin production at $24 \mathrm{~h}$. This had been determined previously and was $25 \mu \mathrm{g}$ of Flamazine $^{\mathrm{TM}} / \mathrm{ml}$ [14]. This was one-quarter of the MIC for strains T1 and T4. Details of the concentrations of each component within the preparation were obtained from the manufacturers and were subject to a confidentiality agreement. The respective concentrations of the individual ingredients were calculated and added to $9 \mathrm{ml}$ of BHI in 20-ml universal bottles. The experiments were set up in triplicate. The ingredients were AgSD, polysorbate 60 , polysorbate 80 , glycerol monostearate, cetyl alcohol, propylene glycol and liquid paraffin. These were incubated at $37^{\circ} \mathrm{C}$ and $200 \mathrm{rpm}$ for $24 \mathrm{~h}$ in an orbital incubator. The viable count and levels of TSST-1 were determined.

\section{Effects of different concentrations of AgSD on TSST-1 production and the effects of agr}

Cultures of strains T1, T4, RN4282 and RN4282agr were grown in BHI supplemented with AgSD 0, 17, 25, 33 and $50 \mu \mathrm{g} / \mathrm{ml}$ for $24 \mathrm{~h}$ in an orbital incubator $\left(37^{\circ} \mathrm{C}\right.$ and $200 \mathrm{rpm}$ ) and viable count and TSST-1 production were determined.

\section{Exoprotein production in subinhibitory concentrations of AgSD}

Overnight broth cultures of $S$. aureus $\mathrm{T} 1$ and $\mathrm{T} 4$ in BHI were washed three times by centrifugation and resuspension in saline and then after the final wash the cells were resuspended in the same volume of fresh BHI; $0.5 \mathrm{ml}$ of a 1 in 100 dilution of this broth was added to $49.5 \mathrm{ml}$ of fresh BHI containing AgSD 25 $\mu \mathrm{g} / \mathrm{ml}$ and incubated at $37^{\circ} \mathrm{C}$ and $200 \mathrm{rpm}$ for $24 \mathrm{~h}$ in an orbital incubator. Samples $(1 \mathrm{ml})$ were removed at 0 , $3,6,24$ and $48 \mathrm{~h}$, the viable count was determined; the remainder was centrifuged at $1500 \mathrm{~g}$ and $4^{\circ} \mathrm{C}$ for $20 \mathrm{~min}$ and the supernatant fluids were stored at $-70^{\circ} \mathrm{C}$

\section{Determination of coagulase activity}

BHI $(100 \mu \mathrm{l})$ was added to each well of a flat-bottomed microtitration plate. Supernatant fluid $(100 \mu 1)$ was added to the first well and doubling dilution was performed by transferring $100 \mu 1$ into the next well and mixing thoroughly. This was repeated for eight wells. 
The final $100 \mu \mathrm{l}$ were discarded. Rabbit plasma (Prolab PL $850 ; 100 \mu \mathrm{l}$ of a $20 \% \mathrm{v} / \mathrm{v}$ dilution in distilled water) was added to each well. The plate was shaken gently to mix the contents, covered and incubated at $37^{\circ} \mathrm{C}$ for $4 \mathrm{~h}$. The titres were recorded as the final well showing a visible clot. Positive and negative controls from previously determined supernates were included in each batch.

\section{Determination of proteolytic activity}

A modification of the method of Lincoln and Leigh [29] was used. Samples of supernatant fluid (100 $\mu \mathrm{l})$ were added to $1 \mathrm{ml}$ of azocasein (Sigma; $1 \mathrm{mg} / \mathrm{ml}$ in $100 \mathrm{~mm}$ Tris- $\mathrm{HCl}, \mathrm{pH} 7.2$ ) and incubated at $37^{\circ} \mathrm{C}$ for $1 \mathrm{~h}$. The reaction was stopped by addition of $1 \mathrm{ml}$ of $5 \% \mathrm{w} / \mathrm{v}$ trichloroacetic acid and mixing; undigested azocasein was allowed to precipitate for $30 \mathrm{~min}$. The mixture was then centrifuged at $10000 \mathrm{~g}$ for $10 \mathrm{~min}$ and the absorbance of the supernate read at $328 \mathrm{~nm}$. One unit of protease activity was defined as giving an absorbance of 0.001 after incubation for $1 \mathrm{~h}$ at $37^{\circ} \mathrm{C}$.

Total protease activity was determined and the activities of serine, thiol and metallo-proteases were determined on the 24-h samples by addition of the appropriate inhibitor and subtraction from the total activity. Serine protease activity was inhibited by addition of $12.5 \mathrm{mM}$ phenyl methyl sulphonyl fluoride (PM SF; Sigma), thiol protease activity was inhibited by addition of $12.5 \mathrm{~mm}$ iodoacetic acid (IAA; Sigma) and metalloprotease activity was inhibited by adding 12.5 $\mathrm{mM}$ ethylenediamine tetraacetic acid (EDTA; Sigma). The inhibitors were incubated with the supernates for $10 \mathrm{~min}$ at $37^{\circ} \mathrm{C}$ before assaying for residual protease activity. The results are the means of three determinations on each sample and the experiment was performed in duplicate.

\section{Statistical analysis}

Where possible, experiments were performed in triplicate and the results were analysed statistically by the unpaired $t$ test with the Minitab program on an IBMcompatible PC.

\section{Results}

\section{Determination of the active component of Flamazine $^{T M}$}

There was an apparent decrease in viable counts at $24 \mathrm{~h}$ when the strains were grown in subinhibitory concentrations of Flamazine ${ }^{\mathrm{TM}}$ or its individual components but these differences were not statistically significant $(p>0.05)$. The results are shown in Table 1. There was an eight-fold increase in TSST-1 in the presence of Flamazine $^{\mathrm{TM}}$ and AgSD and a two-fold increase in the presence of polysorbate 60 or 80 when compared with the levels in BHI alone. These differences were also
Table 1. The effects of the individual components of Flamazine $^{\mathrm{TM}}$ on TSST-1 production in $S$. aureus strain T4

\begin{tabular}{lcc}
\hline Component & $\begin{array}{c}\text { Mean TSST-1 } \\
\text { toxin (units) }\end{array}$ & $\begin{array}{c}\text { Specific TSST-1 } \\
\text { (units } / 10^{9} \\
\text { cfu/ml) }\end{array}$ \\
\hline Control (BHI only) & 16 & 5.1 \\
Flamazine & 128 & $76.6^{*}$ \\
Silver sulphadiazine & 128 & $66.7^{*}$ \\
Polysorbate 60 & 32 & $21.1^{*}$ \\
Polysorbate 80 & 32 & $14.5^{*}$ \\
Glycerol monosterate & 16 & 8.8 \\
Cetyl alcohol & 16 & 8.8 \\
Propylene glycol & 16 & 7.7 \\
Liquid paraffin & 8 & 3.6 \\
\hline
\end{tabular}

Specific toxin production is expressed as units of toxin $/ 10^{9}$ cells $/ \mathrm{ml}$ after $24 \mathrm{~h}$.

$* \mathrm{p}<0.05$.

reflected when TSST-1 production was expressed as specific toxin produced $\left(\mathrm{ng} / 10^{9} \mathrm{cfu} / \mathrm{ml}\right.$; Table 1$)$.

\section{The effects of different concentrations of $\mathrm{AgSD}$ and the effect of agr}

Table 2 shows the response of $S$. aureus strains T1, T4, RN4282 and RN4282agr to different concentrations of $\mathrm{AgSD}$ and the results are expressed as both a titre of TSST-1 and as specific TSST-1, i.e., ng of toxin $/ 10^{9}$ $\mathrm{cfu} / \mathrm{ml}$. AgSD stimulated TSST-1 production in strain $\mathrm{T} 4$ at concentrations of $\geqslant 20 \mu \mathrm{g} / \mathrm{ml}$. There was no effect on the TSST-1 titre in cultures of strain T1, although specific TSST-1 was slightly raised. AgSD also stimulated TSST-1 production in strain RN4282 and, as expected, TSST-1 was greatly reduced in strain RN4282agr but AgSD restored TSST-1 production to almost wild-type levels.

\section{The effects of AgSD on the production of enterotoxins $A$ and $C$}

Both strains $\mathrm{T} 1$ and $\mathrm{T} 4$ produced enterotoxin $\mathrm{A}$ but only T4 produced enterotoxin C. In strain T1, enterotoxin A was detected at $6 \mathrm{~h}$ in the control broth (8 units) whereas in the presence of AgSD, it was detected only at $24 \mathrm{~h}$. There was no difference in the amounts of enterotoxin A produced at $24 \mathrm{~h}$ in the presence of AgSD (64 units) but there was a slight increase (two-fold) at $48 \mathrm{~h}$. In strain $\mathrm{T} 4$ there was no difference in the timing or final amount of enterotoxin A produced.

Strain T4 also produced enterotoxin C. AgSD did not affect the final amount produced when compared to the control.

\section{The effects of AgSD on the production of coagulase}

In strain $\mathrm{T} 1$, coagulase was detected at $3 \mathrm{~h}$ (12 units), increasing to 16 units at $6 \mathrm{~h}$ and reaching a maximum of 64 units at $24 \mathrm{~h}$ and $48 \mathrm{~h}$. In the presence of $\mathrm{AgSD}$, coagulase was detected only at $24 \mathrm{~h}$ and there were no 
Table 2. The effect of different concentrations of AgSD on TSST-1 production in S. aureus T1, T4, RN4282 and RN 4282agr

\begin{tabular}{|c|c|c|c|c|}
\hline \multirow{2}{*}{$\begin{array}{l}\text { Concentration } \\
\text { of } \mathrm{AgSD} \\
(\mu \mathrm{g} / \mathrm{ml})\end{array}$} & \multicolumn{4}{|c|}{ TSST-1 (specific TSST-1) production by $S$. aureus strains } \\
\hline & $\mathrm{T} 1$ & $\mathrm{~T} 4$ & RN4282 & RN4282agr \\
\hline 0 & $128(85.3)$ & $128(34.1)$ & $256(341)$ & $16(17.2)$ \\
\hline 17 & $128(118.5)$ & $128(26.9)$ & $2046(2153)$ & $128(284.4)$ \\
\hline 20 & $256(204.8)$ & $1024(240.9)$ & $2046(2046)$ & $128(232)$ \\
\hline 25 & $128(128)$ & $1024(195)$ & $1024(731.4)$ & $192(240)$ \\
\hline 33 & $256(205)$ & $1024(1024)$ & $1024(2925)$ & $64(160)$ \\
\hline 50 & $256(85.3)$ & 1024 & $1024(1625)$ & $24(36.9)$ \\
\hline
\end{tabular}

Specific toxin (in brackets) is expressed as $\mathrm{ng} / 10^{9}$ cells. The results are expressed as the mean of three experiments. The MIC of AgSD for all strains was $100 \mu \mathrm{g} / \mathrm{ml}$.

differences in the levels produced. Strain T4 produced much lower levels of coagulase and it was first detected at $24 \mathrm{~h}$ (4 units). The levels increased to a maximum at $48 \mathrm{~h}$ (16 units). AgSD had no effect on the timing or levels of coagulase production in strain T4.

\section{The effects of AgSD on the production of proteolytic enzymes}

Fig. 1 shows the effects of AgSD on total protease production. Protease activity was detected in both strains at $3 \mathrm{~h}$ and the levels increased gradually to a maximum at $24 \mathrm{~h}$. In the presence of $\mathrm{AgSD}$, protease production was delayed but there was no significant difference $(p>0.05)$ at $24 \mathrm{~h}$ when compared to the control broth. Strain T4 produced twice as much total protease when compared with strain $\mathrm{T} 1$. The activities of the individual proteases after $24 \mathrm{~h}$ are shown in Fig. 2 . The predominant protease produced by strain $\mathrm{T} 1$ was thiol protease at $72 \%$ of the total activity followed by serine protease $(20 \%)$ and metalloprotease $(8 \%)$. In the presence of AgSD there was little difference from the control culture. Strain T4 produced two-fold higher protease and the predominant activity was metalloprotease $(74 \%)$ followed by serine protease $(23 \%)$ and thiol protease $(3 \%)$. In the presence of AgSD the total protease activity was similar to the control but the relative proportions of the different activities were altered with serine and thiol-proteases being dominant
(52\% and $47 \%$, respectively) and metalloprotease activity was greatly reduced $(2 \%)$.

\section{Discussion}

The results obtained confirm that the marked increase in TSST-1 production in $S$. aureus strain $\mathrm{T} 4$ by subinhibitory concentrations of Flamazine ${ }^{\mathrm{TM}}$ was due to the active ingredient AgSD. The increase in TSST-1 production caused by AgSD also occurred in strains RN4282 and RN4282agr and was, therefore, independent of agr. Studies on the effects of AgSD on the other exoproteins produced by strains $\mathrm{T} 1$ and $\mathrm{T} 4$ showed that the effects of AgSD were dependent on the strain and the exoprotein. Strain T1 was originally isolated from a menstrual case of TSS and it is probable that the array of expressed virulence factors differs from strain T4, which was isolated from a patient with TSS resulting from an infected burn wound. Of the exoproteins studied here, strain T1 differed from $\mathrm{T} 4$ in the variety of proteases produced and strain $\mathrm{T} 4$ also produced enterotoxin $\mathrm{C}$.

AgSD had no effect on enterotoxin $\mathrm{A}$ and $\mathrm{C}$ production in strain $\mathrm{T} 4$ and delayed enterotoxin A production in strain T1 without a marked effect on levels. AgSD had no effect on coagulase production by either strain. In this study, only one subinhibitory concentration of AgSD was used to determine if it had any global

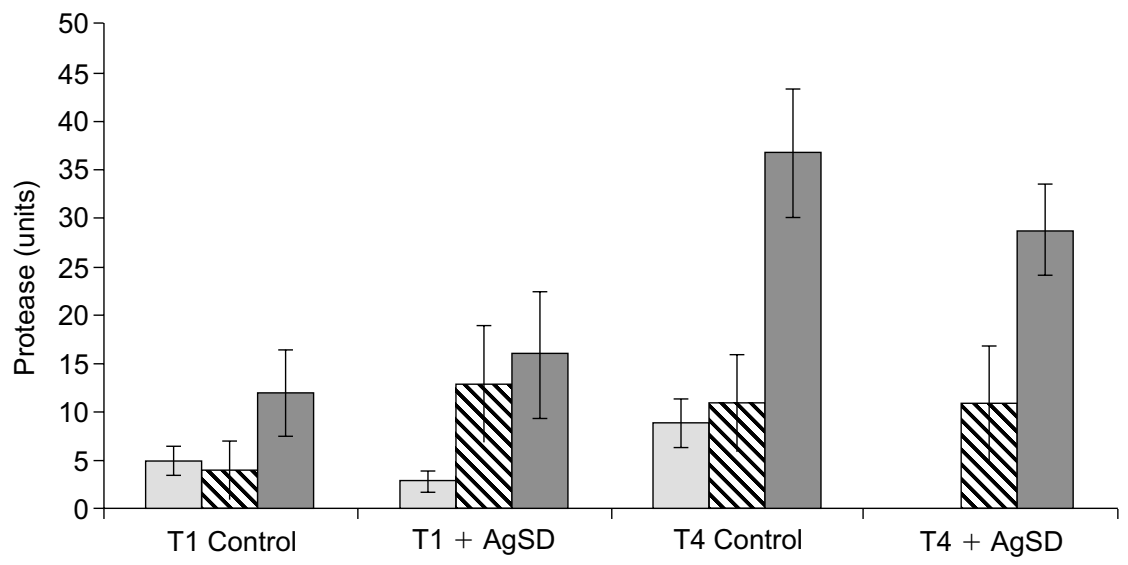

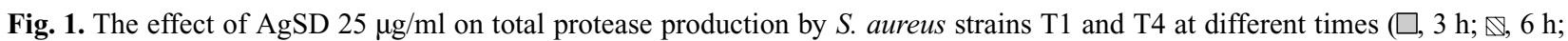
$\square, 24 \mathrm{~h}$ ); Control = BMI only. 


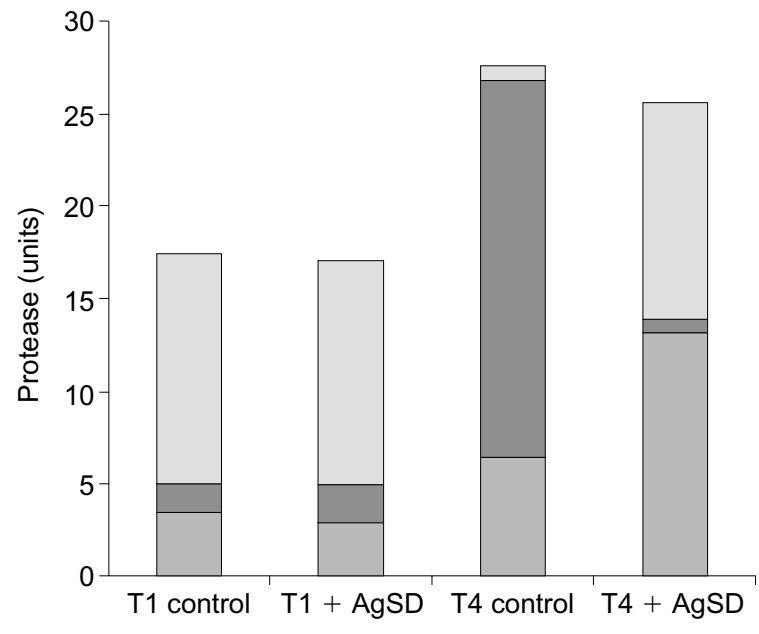

Fig. 2. The effect of AgSD $25 \mu \mathrm{g} / \mathrm{ml}$ on individual proteases produced by $S$. aureus strains T1 and T4 after $24 \mathrm{~h} ; \square$ thiol protease; $\square$, metalloprotease; $\square$, serine protease; control $=$ BHI only.

effects on other toxins or just affected TSST-1. AgSD appeared to markedly increase TSST-1 only in certain strains while other toxins were not affected.

AgSD increased TSST-I production significantly in strain T4 and the reason for this is unknown and requires further investigation. Transcription of tst is under the control of agr [9]. The effector molecule, RNAIII, is synthesised in mid-to-late exponential growth phase as a result of induction by an autocrine pheromone octapeptide [6]. RNAIII induces transcription of $t s t$ by an unknown mechanism but tst mRNA does not appear until one or two generations after RNAIII synthesis [30]. Expression of agr and tst is also affected by sarA, another regulatory molecule, which is produced in response to microaerobic conditions [31]. Production of TSST-1 toxin has been shown previously to occur earlier in the growth cycle in the presence of Flamazine ${ }^{\mathrm{TM}}[14]$ and the same effect has been observed with AgSD (unpublished data). Why this earlier expression of TSST-1 should occur is unknown but these observations, alongside the increased TSST-1 levels observed in the agr mutant, suggest that $\mathrm{AgSD}$ does not affect agr regulation. Production of enterotoxin A, which is also co-ordinately regulated by agr, was not affected, again suggesting that the effect was independent of agr. It could be postulated that the earlier expression of tst might account for the increased levels of TSST-1. However, comparison of TSST-1 production at $48 \mathrm{~h}$ and $72 \mathrm{~h}$ (data not shown) still showed a four-fold increase in the presence of AgSD compared with the control broth.

It is known that silver ions react with both proteins and nucleic acids. It is possible that $\mathrm{Ag}^{2+}$ may cause distortion to DNA and allow activation of genes such as $t s t$ by binding to the DNA and facilitating RNApolymerase binding. Studies have shown that when $S$. aureus is grown in subinhibitory concentrations of novobiocin, known to cause conformational changes to
DNA, increased levels of eta are produced [32]. In attempts to isolate DNA from AgSD-treated cells and, with increasing AgSD concentrations, the DNA becomes increasingly yellow in colour and its solubility is also affected (unpublished observations). The inhibition of TSST-1 production caused by the agr mutation in strain RN4282agr was effectively reversed in the presence of AgSD, again suggesting an interaction with the DNA.

Other possibilities for TSST-1 being increased in the presence of AgSD include enhanced stability of tst mRNA, although it would not explain the earlier appearance of TSST-1 in the growth cycle. Previous workers have shown that metals, including $\mathrm{Ag}^{2+}$, increased the resistance of tobacco mosaic virus RNA to ribonuclease without loss of infectivity [33]. The effects of AgSD on tst mRNA and RNAIII are currently being investigated. Another possibility is at the level of translation and more detailed studies need to be undertaken to unravel the possible effect(s). It also may be possible that AgSD enhances secretion of TSST-1 although there is no evidence that TSST-1 is stored intracellularly before excretion. The protein is translated from the mRNA as a pro-toxin with a 40 amino acid N-terminal signal peptide that is removed during transport across the membrane [34]. Finally, inhibition of the degradation of toxin by proteolytic enzymes could enhance the amount of toxin present in the supernate by inhibiting turnover. Other workers have shown that $S$. aureus isolates from cases of MTSS produced thiol protease (type II) as their major class of protease [35]. Strains that produced predominantly serine protease (type I) were not associated with cases of TSS [36]. Serine protease was later shown to digest TSST-1 [37] and it was postulated that strains producing this as a predominant protease produced little or no TSST-1. In this study, strain T1 produced predominantly thiol protease and strain $\mathrm{T} 4$ produced predominantly metalloprotease. AgSD did not affect total protease but inhibited metalloprotease and increased thiol and serine proteases. It has been shown recently that different proteases are responsible for cleavage of different exoproteins and Chan and Foster [31] suggest a major role for proteases in modulation of virulence determinants. For example, metalloprotease activates serine protease by proteolytic cleavage [38] and the serine protease modifies surface-bound proteins [39]. Furthermore, a role has been suggested for proteases in the activation of exocellular lipases in $S$. hyicus [40,41] and $S$. aureus [42]. The results showed that production of metalloprotease was greatly reduced by AgSD in strain T4 but not T1. This might suggest a role for metalloprotease in the turnover of TSST-1. Chan and Foster [8] showed that incorporation of EDTA enhanced TSST-1 production by stimulating tst expression. Whether or not this was due to inhibition of metalloprotease by EDTA was not fully investigated. Further investigations are needed to determine whether other metalloprotease inhibitors have a 
similar effect, such as 1,10-phenanthroline, would be worth trying. It has been shown that there are at least five proteases in S. aureus 8325-4 [31] and further work is required to determine whether AgSD specifically affects any or all these.

Overall, the results show that the effects of AgSD are strain dependent and the differences between responder and non-responder strains are being investigated.

We thank R. P. Novick and T. J. Foster for gifts of strains and Oxoid for supply of toxin assay kits at reduced cost.

\section{References}

1. Hiramatsu K, Aritaka N, Hanaki $\mathrm{H}$ et al. Dissemination in Japanese hospitals of strains of Staphylococcus aureus heterogenously resistant to vancomycin. Lancet 1997; 350: 1670-1673.

2. Chesney PJ. Toxic shock syndrome. In: Crossley KB, Archer GL (eds) The staphylococci in human disease. New York, Churchill Livingstone. 1997: 509-525.

3. Waldvogel FA. Staphylococcus aureus (including toxic shock syndrome). In: Mandell GL, Bennett JE, Dolin R (eds) Principles and practice of infectious diseases, 4th edn, vol 2. New York, Churchill Livingstone. 1995: 1754-1777.

4. Foster TJ, Höök M. Surface protein adhesins of Staphylococcus aureus. Trends Microbiol 1998; 6: 484-488.

5. Sarafian SK, Morse SA. Environmental factors affecting toxic shock syndrome toxin-1 (TSST-1) synthesis. J Med Microbiol 1987; 24: 75-81.

6. Ji G, Beavis RC, Novick RP. Cell density control of staphylococcal virulence mediated by an octapeptide pheromone. Proc Natl Acad USA 1995; 92: 12055-12059.

7. Reeves MW. Effect of trace metals on the synthesis of toxic shock syndrome toxin 1. Rev Infect Dis 1989; 11 Suppl 1: S145-S150.

8. Chan PF, Foster SJ. The role of environmental factors in the regulation of virulence-determinant expression in Staphylococcus aureus 8325-4. Microbiology. 1998; 144: 2469-2479.

9. Recsei P, Kreiswirth B, O'Reilly M, Schlievert P, Gruss A, Novick RP. Regulation of exoprotein gene expression in Staphylococcus aureus by agr. Mol Gen Genet 1986; 202: 58-61.

10. Schlievert PM, Kelly JA. Clindamycin-induced suppression of toxic-shock syndrome-associated exotoxin production. $J$ Infect Dis 1984; 149: 471 .

11. Andrews MM, Giacobbe KD, Parsonnet J. Induction of toxic shock syndrome toxin-1 (TSST-1) and beta-lactamase by subinhibitory concentrations of beta-lactam antibiotics. Biomedicine 1996, Washington DC, May 3-6, 1996, Abstract N-IN0011.

12. Projan SJ, Brown-Skrobot S, Schlievert PM, Vandenesch F, Novick RP. Glycerol monolaurate inhibits the production of beta-lactamase, toxic shock syndrome toxin-1, and other staphylococcal exoproteins by interfering with signal transduction. J Bacteriol 1994; 176: 4204-4209.

13. Schlievert PM, Deringer JR, Kim MH, Projan SJ, Novick RP. Effect of glycerol monolaurate on bacterial growth and toxin production. Antimicrob Agents Chemother 1992; 36: 626-631.

14. Edwards-Jones V, Foster HA. The effects of topical antimicrobial agents on the production of toxic shock syndrome toxin-1. J Med Microbiol 1994; 41: 408-413.

15. Wruble M. Colloidal silver sulphonamides. J Am Pharm Assoc 1943; 32: 80-82.

16. Fox CL. Silver sulphadiazine - a new topical therapy for Pseudomonas in burns. Arch Surg 1968; 96: 184-188.

17. Fox CL, Rappole BW, Stanford W. Control of pseudomonas infection in burns by silver sulphadiazine. Surg Gynecol Obstet 1969; 128: 1021-1026.

18. Fox CL, Modak SM. Mechanism of silver sulphadiazine action on burn wound infections. Antimicrob Agents Chemother 1974; 5: $582-588$

19. Wysor MS, Zollinhofer RE. On the mode of action of silver sulphadiazine. Pathol Microbiol 1972; 38: 296-308.

20. Bult A. Silver sulphadiazine and related antibacterial metal sulphanilamides: facts and fancy. Pharm Int 1982; 3: 400-404.

21. Hamilton-Miller JMT, Shah S, Smith C. Silver sulphadiazine: a comprehensive in vitro reassessment. Chemotherapy 1993; 39: 405-409.

22. Bridges K, Lowbury EJL. Drug resistance in relation to use of silver sulphadiazine cream in a burns unit. J Clin Pathol 1977; 30: $160-164$.

23. Rosenkranz HS, Rosenkranz S. Silver sulphadiazine: interaction with isolated deoxyribonucleic acid. Antimicrob Agents Chemother 1972; 2: 373-383.

24. Rosenkranz HS, Carr HS. Silver sulphadiazine: effect on the growth and metabolism of bacteria. Antimicrob Agents Chemother 1972; 2: 367-372.

25. Jensen RH, Davidson N. Spectrophotometric, potentiometric, and density gradient ultracentrifugation studies of the binding of silver ion by DNA. Biopolymers 1966; 4: 17-32.

26. Coward JE, Carr HS, Rosenkranz HS. Silver sulphadiazine: effect on the growth and ultrastructure of staphylococci. Chemotherapy 1973; 19: 348-353.

27. Richards RME, Taylor RB, Xing DKL. An evaluation of antibacterial activities of combinations of sulphonamides, trimethoprim, dibromopropamidine, and silver nitrate compared with their uptakes by selected bacteria. J Pharmaceut Sci 1991; 80: 861-867.

28. Miles AA, Misra SS. The estimation of the bactericidal power of the blood. J Hyg 1938; 38: 732-748.

29. Lincoln RA, Leigh JA. Production of extracellular proteases from Staphylococcus aureus strain V8 in dialysis culture. J Microbial Methods 1994; 20: 175-182.

30. Taylor D, Arvidson S. Lack of correlation between the levels, and time of appearance, of TSST-1 and hld mRNAs under certain growth conditions of $S$. aureus. Zentralbl Bakteriol Suppl 1994; 26: 195-200.

31. Chan PF, Foster SJ. Role of SarA in virulence determinant production and environmental signal transduction in Staphylococcus aureus. J Bacteriol 1998; 180: 6232-6241.

32. Sheenan BJ, Foster TJ, Dorman CJ, Park S, Stewart GSAB Osmotic and growth-phase dependent regulation of the eta gene of Staphylococcus aureus: a role for supercoiling. Mol Gen Genet 1992; 232: 49-57.

33. Singer B, Fraenkel-Conrat H. Enzyme resistance of complexes with ribonucleic acid with metals. Biochemistry 1962; 1: $852-858$.

34. Arvidson S. Regulation of synthesis of TSST-1. In: Arbuthnott J, Furman B (eds) European conference on toxic shock syndrome. International Congress and Symposium Series 229. London, Royal Society of Medicine Press. 1998: 101-106.

35. Todd JK, Franco-Buff AM, Lawellin DW, Vasil ML. Distinctive protease activity of TSS associated Staphylococcus aureus strains. Zentralbl Bakteriol Suppl 1985; 14: 111-113.

36. Todd JK. Toxic shock syndrome. Clin Microbiol Rev 1988; 1: 432-446.

37. Lawellin DW, Franco-Buff AM, Todd JK. Alteration of proteins reacting with antibody to toxic shock syndrome toxin-1 by endogenous proteases of Staphylococcus aureus. Rev Infect Dis 1989; 11 Suppl 1: S117-S123.

38. Drapeau GR. Role of a metalloprotease in activation of the precursor of protease. J Bacteriol 1978; 136: 607-613.

39. McGavin MJ, Zahradka C, Rice K, Scott JE. Modification of the Staphylococcus aureus fibronectin binding phenotype by V8 protease. Infect Immun 1997; 65: 2621-2628.

40. Ayora S, Gotz F. Genetic and biochemical properties of an extracellular neutral metalloprotease from Staphylococcus hyicus subsp. hyicus. Mol Gen Genet 1994; 242: 421-430.

41. Ayora S, Lindgren PE, Gotz F. Biochemical properties of a novel metalloprotease from Staphylococcous hyicus subsp. hyicus involved in extracellular lipase processing. $J$ Bacteriol 1994; 176: 3218-3223.

42. Rollof J, Normark S. In vivo processing of Staphylococcus aureus lipase. J Bacteriol 1992; 174: 1844-1847. 